\title{
A NOTE ON A PROBLEM OF SAFF AND VARGA CONCERNING THE DEGREE OF COMPLEX RATIONAL APPROXIMATION TO REAL VALUED FUNCTIONS
}

\author{
BY S. W. ELLACOTT ${ }^{1}$
}

Saff and Varga [1977, 1978] discovered the surprising fact that given a real valued function on $[-1,1]$ one can sometimes obtain a better rational approximation to $f$ of a given type by allowing complex coefficients than by restricting the coefficients to be real. In this note we point out a connection between this result and the "Trefethen effect" (Trefethen [1981a, 1981b]) of near circular error curves for best approximation on the unit disc, which as Trefethen has shown is in turn closely related to the Carathéodory-Fejér Theorem and its generalisation to meromorphic approximation due to Takagi.

We consider a compact simply connected set $\Omega$ in the complex plane which is symmetric about the real line (i.e. $z \in \Omega$ iff $\bar{z} \in \Omega$ ). We assume further that the complement of $\Omega$ is simply connected in the extended plane and that $\Omega$ is a Faber domain (see e.g. Gaier [1980]). Finally if $\psi$ denotes the conformal mapping of the complement of the unit disc $D$ onto the complement of $\Omega$ such that $\psi(\infty)=\infty, \lim _{w \rightarrow \infty} \psi(w) / w$ finite real and positive, we assume for simplicity that $\psi$ can be extended continuously to the boundary of the disc. All these properties are, of course, satisfied for the main case of interest here, $\Omega=$ $I=[-1,1]$.

$\mathfrak{A}(\Omega)$ will denote the set of functions continuous on $\Omega$ and analytic at interior points, and $\mathfrak{U}^{R}(\Omega)$ the subspace of functions satisfying $f(z)=\overline{f(z)}$. $\mathfrak{I}: \mathfrak{U}(D) \rightarrow \mathfrak{U}(\Omega)$ is the Faber transform (Gaier [1980]); $E_{m n}^{C}(f ; \Omega)$ the error of best (Chebyshev) approximation to $f \in \mathfrak{A}^{R}(\Omega)$ from $\Re_{m n}(\Omega)$, the set of type $(m, n)$ rational functions with no poles in $\Omega$, and $E_{m n}^{R}(f ; \Omega)$ the corresponding error of best approximation from the subset of rational approximations with real coefficients.

Saff and Varga $[1977,1978]$ observed that it is possible to find $f \in \mathfrak{A}^{R}(I)$ for which $E_{n n}^{C}(f ; I)<E_{n n}^{R}(f ; I)$ (e.g. $\left.f(x)=x^{2}, n=1\right)$. Some authors attacked the case $n=1$ by largely geometric arguments and showed among other things

Received by the editors July 14, 1981.

1980 Mathematics Subject Classification. Primary 30E10; Secondary 41 A20, 41 A25, $41 \mathrm{~A} 50$.

${ }^{1}$ Author partially supported by a Royal Society European Visiting Fellowship. 
that for certain classes of functions $E_{11}^{C}(f ; I) / E_{11}^{R}(f ; I) \geqslant 1 / 2$ (Ruttan [1977]; Bennet, Rudnick and Vaaler, [1979]). We consider more generally $m \geqslant n-1$ and obtain a lower bound for $E_{m n}^{C}(f ; \Omega) / E_{m n}^{R}(f ; \Omega)$ which, if $m \geqslant n$ and $\Omega$ is convex, is for many functions very close to $1 / 2$.

We introduce the space $\widetilde{\Re}_{m n}(D)$ which may be described as those functions of the form

$$
r(z)=\frac{\sum_{j=-\infty}^{m} a_{j} z^{j}}{\sum_{j=0}^{n} b_{j} z^{j}}
$$

where the denominator has no zeros in $D$ and the numerator represents a function bounded on $S=\{z|| z \mid=1\}$ and analytic on the complement of $D$. Define $\widetilde{E}_{m n}^{R}(f ; S)$ and $\widetilde{E}_{m n}^{C}(f, S)$ for $f \in \mathfrak{Q}^{R}(D)$ in the obvious way.

We now list some simple relationships which are either obvious or straightforward consequences of results given in Trefethen [1981b], Gutknecht [1981] or Ellacott [1981]. Here $g \in \mathfrak{A}^{R}(D)$.

(i) $\widetilde{E}_{m n}^{R}(g ; S)=\widetilde{E}_{m n}^{C}(g ; S)$. From now on we drop the superscripts $R$ and $C$ when referring to this error,

(ii) $\mathfrak{I}(g) \in \mathfrak{A}^{R}(\Omega)$.

(iii) $\widetilde{E}_{m n}(g ; S) \leqslant E_{m n}^{C}(g ; D) \leqslant E_{m n}^{R}(g ; D)$ and for many "reasonable" functions $g$ the ratio $\rho(g)=E_{m n}^{R}(g ; D) / \widetilde{E}_{m n}(g ; S) / E_{m n}^{R}(g ; D)$ is very close to 1 .

In particular equality holds if $g$ is a polynomial of degree $m+1$.

(iv) If $m \geqslant n-1, \widetilde{E}_{m n}(g ; S) \leqslant E_{m n}^{C}(\mathfrak{I}(g) ; \Omega) \leqslant E_{m n}^{R}(\mathcal{I}(g) ; \Omega)$.

(v) If $m \geqslant n-1, E_{m n}^{P}(\mathcal{I}(g) ; \Omega) \leqslant T E_{m n}^{P}(g ; D)$, where $P=R$ or $C$ and $T=\|\mathfrak{I}\|$ for $m=n-1$ or $\left\|\mathfrak{I}_{0}\right\|$ for $m \geqslant n$, where $\mathfrak{I}_{0}$ is defined by $\mathfrak{I}_{0}(g)=$ $\mathfrak{I}(g)+g(0)$. For convex $\Omega,\|\mathfrak{I}\| \leqslant 5$ and $\|\mathfrak{I}\| \leqslant$,2 .

Unfortunately it is not entirely clear at present what a "reasonable" function in the sense of (iii) is but it appears to have some connection with the regularity of the Taylor coefficients. (Trefethen [1981b], has some asymptotic results and some numerical computations for $e^{z}$, and further numerical results are given in Ellacott [1981]). Not every $f \in \mathfrak{A}^{R}(\Omega)$ is of the form $\mathfrak{T}(g), g \in$ $\mathfrak{A}^{R}(D)$, but for any function which can be so expressed (e.g. any polynomial or any function with a uniformly and absolutely convergent Faber series) and for $m \geqslant n-1$ (iv) and (v) yield immediately

$$
\frac{E_{m n}^{C}(f ; \Omega)}{E_{m n}^{R}(f ; \Omega)} \geqslant \frac{\rho\left(\mathfrak{T}^{-1}(f)\right)}{T} \text {. }
$$

In particular,

(1a) $E_{n-1, n}^{C}(f ; I) / E_{n-1, n}^{R}(f ; I) \geqslant \rho\left(\mathfrak{I}^{-1}(f)\right) / 5$.

(1b) $E_{m n}^{C}(f ; I) / E_{m n}^{R}(f ; I) \geqslant \rho\left(\mathfrak{T}^{-1}(f)\right) / 2, m \geqslant n$. 
If $f$ is a polynomial of degree $m+1$ we have

(2) $E_{m n}^{C}(f ; I) / E_{m n}^{R}(f ; I) \geqslant 1 / 2, m \geqslant n$, and for "reasonable" functions $f$ (i.e. functions for which $\mathfrak{I}^{-1}(f)$ is reasonable in the sense of (iii)), we would not expect the lower bounds given by (1a) and (1b) to be much less than $1 / 5$ or $1 / 2$ respectively.

Similar considerations hold for the problem of approximation by the real parts of rational functions as considered by Wulbert [1978].

We conclude with three questions suggested by these remarks. Firstly, and most obviously, is $1 / 2$ actually a lower bound for $E_{m n}^{C}(f ; I) / E_{m n}^{R}(f ; I)$ for $f \in$ $\mathfrak{A}^{R}(I)$ and $m \geqslant n$, and, if so, is it sharp?

The second question is related: Are there any functions in $\mathfrak{A}^{R}(D)$ for which $E_{m n}^{C}(f ; D)<E_{m n}^{R}(f ; D)$ ? If so, they are likely to be more difficult to find than for $I$; (2) shows that $f(z)=z^{2}$ with $m=n=1$ will not do. The third question is more general: Certain asymptotic results are known about the behaviour of Faber polynomials as the degree $\rightarrow \infty$ (Pommerenke $[1964,1967]$ ). Can these be applied to discuss the asymptotic behaviour of $E_{m n}^{C}(f ; \Omega) / E_{m n}^{R}(f ; \Omega$

\section{REFERENCES}

[1979] C. Bennet, K. Rudnick and J. Vaaler, Best uniform approximation by linear fractional transformations, J. Approximation Theory, 25, 204-224.

[1981] S. Ellacott, On the Faber transform and efficient numerical rational approximation, Forschungsinstitut für Mathematik, ETH Zürich, SIAM J. Numer. Anal. (submitted)

[1980] D. Gaier, Vorlesungen über Approximation im Komplexen, Birkhäuser, Basel.

[1981] M. Gutknecht, Rational CF approximation on a disc, on a circle and on an interval, Seminar für Angewandte Mathematik, ETH Zürich (in preparation).

[1964] Ch. Pommerenke, Ueber die Faberschen Polynome Schlichter Funktionen, Math. Z. 85, 197-208.

[1967] - Konforme Abbildung und Fekete-Punkte, Math. Z. 89, 422-438.

[1977] A. Ruttan, On the cardinality of a set of best complex rational approximations to a real function, Pade' and Rational Approximation (E. B. Saff and R. S. Varga, eds.), Academic Press, New York, pp. 303-319.

[1977] E. B. Saff and R. S. Varga, Nonuniqueness of best approximating complex rational functions, Bull. Amer. Math. Soc. 83, 375-377.

[1978] Nonuniqueness of best complex rational approximations to real functions on real intervals, J. Approximation Theory, 23, 78-85.

[1981a] L. Trefethen, Near circularity of the error curve in complex Chebyshev approximation, J. Approximation Theory, 31, 344-366.

[1981b] Rational Chebyshev approximation on the unit disc, Numer. Math. 37, 297-320.

[1979] L. Trefethen, Near circularity of the error curve in complex Chebyshev approximation, Computer Science Dept., Stanford Univ., J. Approximation Theory (to appear).

[1980] , Rational Chebyshev approximation on the unit disc, Stanford NAP manuscript NA-80-08, Stanford Univ., California, Numer. Math. (submitted).

[1978] D. Wulbert, The rational approximation of real functions, Amer. J. Math. vol. $100,1281-1315$. ENGLAND

DEPARTMENT OF MATHEMATICS, BRIGHTON POLYTECHNIC, BRIGHTON, 